\title{
Avaliação do Programa de Tuberculose em Sapucaia do Sul (RS): indicadores, 2000-2008
}

\author{
Evaluation of the tuberculosis program in Sapucaia do Sul, \\ state of Rio Grande do Sul: indicators, 2000-2008
}

Maria Antonia Heck ${ }^{1}$

Juvenal Soares Dias da Costa ${ }^{2}$

Marcelo Felipe Nunes ${ }^{3}$

\footnotetext{
${ }^{1}$ Vigilância

Epidemiológica, Secretaria Municipal de Saúde, Prefeitura Municipal de Sapucaia de Sul. Rua Leônidas de Sousa 999, Três Portos. 93210-140 Sapucaia do Sul RS. maria.heck@gmail.com ${ }^{2}$ Departamento de Medicina Social, Faculdade de Medicina, Universidade Federal de Pelotas. ${ }^{3}$ Programa de PósGraduação em Saúde Coletiva, Universidade do Vale do Rio dos Sinos.
}

\begin{abstract}
The scope of this study was to describe the trends of tuberculosis indicators in relation to their prevalence, incidence and the case outcome percentages (cure, abandonment of treatment or death) for the patients who entered in the Program in Sapucaia do Sul, state of Rio Grande do Sul, between 2000 and 2008. An ecological analysis of the incidence was conducted and the gross frequency rates and respective confidence intervals of $95 \%$ were presented. The rate estimates were verified by means of average annual percent change as well as the mobile averages every three years. The prevalence rate was 64.3 and the incidence rate was 58.0 per 100.000 inhabitants. Anal$y$ sis of the indicators did not reveal statistically significant differences between the annual rates or in the mobile averages during the period. The outcomes were below the targets proposed by the World Health Organization, what compromises control of the disease, as it is not proving possible to interrupt the chain of transmission.
\end{abstract}

Key words Tuberculosis, Prevalence, Incidence, Mortality, Following medication instructions
Resumo Objetivo: descrever a tendência dos indicadores da tuberculose em relação à sua prevalência e incidência e os percentuais dos desfechos dos casos no encerramento (cura, abandono ao tratamento ou óbito) dos pacientes que ingressaram no Programa em Sapucaia do Sul, RS, entre 2000 e 2008. Métodos: Foi realizada uma análise ecológica das taxas. Foram apresentadas as taxas brutas de frequência e os respectivos intervalos de confiança a 95\%. Verificaram-se as estimativas das taxas através das variações percentuais médias anuais. Foram realizadas médias móveis a cada três anos. Resultados: As taxas médias de prevalência e incidência foram de 64,3 e 58,0 por 100.000 habitantes respectivamente. As análises dos indicadores não apresentaram diferenças estatisticamente significativas entre as taxas anuais ou nas médias móveis durante o período. Discussão: Os desfechos estavam abaixo das metas propostas pela Organização Mundial de Saúde o que compromete o controle da doença, já que não se consegue interromper a cadeia de transmissão.

Palavras-chave Tuberculose, Prevalência, Incidência, Mortalidade, Adesão à medicação 


\section{Introdução}

A tuberculose é uma doença com formas de transmissão e de prevenção conhecidas, com critérios diagnósticos bem definidos, sendo curável em praticamente $100 \%$ dos casos novos através de tratamento padronizado e oferecido para todos os acometidos no sistema de saúde público brasileiro. Apesar dessa disponibilidade ainda apresenta-se como problema de saúde pública, pela dificuldade de se alcançar as metas de controle ${ }^{1-3}$

A Organização Mundial da Saúde (OMS) considerou a tuberculose em estado de emergência no mundo, por ser ainda a maior causa de morte por doença infecciosa em adultos. Dois bilhões de pessoas (um terço da população mundial) estão infectadas pelo Mycobacterium tuberculosis. Destes, oito milhões desenvolverão a doença e dois milhões morrerão a cada ano. Diante deste cenário internacional a Organização Mundial da Saúde vem propondo estratégias globais buscando a diminuição e o controle da doença ${ }^{4-6}$. O Brasil ocupa o $18^{\circ}$ lugar entre os 22 países responsáveis por $80 \%$ do total de casos de tuberculose no mundo. Desde 2003 a tuberculose tem sido colocada na agenda de prioridade de políticas públicas do Brasil e o Ministério da Saúde (MS) tem pactuado com as demais esferas de gestão o fortalecimento da estratégia DOTS (Directly Observed Therapy Short-Course - Tratamento diretamente supervisionado de curta duração). Trata-se do principal instrumento para alcançar as metas nacionais e internacionais com o objetivo de reduzir pela metade a incidência da tuberculose no país até o ano de 2015: diagnosticar pelo menos $70 \%$ dos casos novos estimados, curar $85 \%$ deles e reduzir o abandono ao tratamento e os óbitos em menos de $5 \%{ }^{7-15}$.

Assim, o objetivo do presente estudo foi descrever a tendência dos indicadores da tuberculose em relação à prevalência e incidência da doença. Foram descritos também os percentuais dos possíveis desfechos dos casos na situação de encerramento (cura, abandono ao tratamento ou óbito) dos pacientes que ingressaram no Programa de Controle de Tuberculose no município de Sapucaia do Sul, RS, entre os anos de 2000 e 2008.

\section{Métodos}

O município de Sapucaia do Sul está localizado na Região Metropolitana de Porto Alegre, no Vale do Rio dos Sinos, no Estado do Rio Grande do
Sul, a população estimada pelo IBGE em 2008 foi de 126.085 habitantes, área de $65,2 \mathrm{Km}^{2}\left(42,1 \mathrm{Km}^{2}\right.$ de área urbana, $23,1 \mathrm{Km}^{2}$ de área rural) e densidade demográfica de 2.084,3 hab./ $\mathrm{Km}^{2}$ A atividade econômica do município advém de 79,5\% da indústria e 20,9\% do comércio, serviços e agricultura $^{16} \mathrm{~A}$ rede municipal de saúde oferece 18 unidades básicas de saúde, sendo 13 com a Estratégia de Saúde da Família. Complementam o sistema cinco unidades de referência secundária, sendo que uma atende portadores de tuberculose e coinfecção TB-HIV, duas em saúde mental, pronto atendimento 24 horas e o Hospital $\mathrm{Mu}-$ nicipal Getúlio Vargas que atende internação por tuberculose e dispõe de raio $\mathrm{X}$, um laboratório credenciado para baciloscopia e outros exames complementares. Também existe uma rede de atenção privada, financiada principalmente através da indústria e comércio local ${ }^{16}$

Foi realizada uma análise ecológica das taxas estabelecidas a partir de dados secundários notificados no Sistema de Informação de Agravos de Notificação (SINAN/SMS) ${ }^{16}$ sobre a tendência dos indicadores de tuberculose no município de Sapucaia do Sul entre 2000 e 2008.

A fonte de dados foi obtida a partir da base do DATASUS/SINAN/SMS, do livro de registros e controle de tratamento de casos de tuberculose e arquivos com as fichas de notificação/investigação de tuberculose.

Os dados do SINAN/SMS foram obtidos através de relatórios operacionais no TABWIN, que contemplou a informação de cura, abandono e óbito no desfecho do caso por ocasião da situação de encerramento. Os óbitos foram verificados semanalmente no Sistema de Informações sobre Mortalidade e não apresentaram inconsistências.

Foram excluídos do estudo os casos notificados no SINAN no período em estudo referentes aos residentes no município de Sapucaia do Sul, porém notificados e tratados em outros municípios; duplicidade (um caso); casos notificados em Sapucaia do Sul, mas moradores em outros municípios.

Neste estudo foram classificados como: "casos de tuberculose” indivíduos com diagnóstico confirmado por baciloscopia ou cultura e aqueles em que o médico, com base nos dados clínico-epidemiológicos e nos exames complementares, firmou o diagnóstico de tuberculose.

Para o cálculo da prevalência anual foram considerados todos os casos incluídos no Programa, independente do tipo de entrada e da forma clínica da doença. 
Para incidência anual utilizaram-se os casos novos notificados no SINAN/SMS. "Casos novos" foram doentes com tuberculose que nunca usaram ou usaram por menos de um mês os medicamentos antituberculose.

A partir dos casos novos anuais foram descritos percentualmente os desfechos possíveis ao final do tratamento: alta por cura, abandono ao tratamento e óbitos por tuberculose.

Os casos de "alta por cura" foram considerados nas seguintes situações: quando, ao completar o tratamento, os pacientes apresentavam duas baciloscopias negativas (cura bacteriológica comprovada); aqueles que tiveram alta com base em dados clínicos e exames complementares (cura clínica não comprovada bacteriologicamente); casos de bacilíferos negativos no início do tratamento ou extrapulmonares quando completavam o tratamento e apresentavam melhora clínica. "Abandono" foram os casos que deixaram de tomar os medicamentos antituberculose por mais de trinta dias consecutivos. "Óbito" foi considerado todo caso que se tomou conhecimento da morte do paciente, durante o tratamento e independente da causa. As altas por falência ao tratamento, toxicidade e transferência, independente da causa, não foram avaliadas ${ }^{2,17-18}$.

Para a elaboração das taxas por 100.000 habitantes foram utilizados os dados populacionais do Instituto Brasileiro de Geografia e Estatística.

A entrada de dados foi realizada através do Excel. As taxas brutas foram analisadas através dos intervalos de confiança a 95\%. O erro padrão das taxas foi estabelecido a partir da fórmula $[\sqrt{ }($ taxa/ população)x1000 ${ }^{19}$ Verificaram-se também as estimativas das taxas através das variações percentuais médias anuais. Utilizaram-se as variações percentuais médias anuais através da fórmula: $\left\{\Sigma\left[\left(\text { Taxa }_{\text {ano i }} / \text { Taxa }_{\text {ano i-1 }}\right)-1\right] \times 100 / \mathrm{n}-1\right\}^{19}$.

Foram realizadas médias móveis, taxas resultantes das combinações dos numeradores e dos denominadores a cada três anos. Esta estratégia pretendeu aumentar a estabilidade dos indicadores, diminuindo sua variação aleatória, pelo aumento do tamanho da amostra em cada ponto de tempo ${ }^{20}$.

Os desfechos possíveis ao final do tratamento foram analisados através do teste Qui-quadrado para tendência no Programa Epi-Info ${ }^{19}$.

O Projeto de Pesquisa foi aprovado pelo Comitê de Ética e Pesquisa da Faculdade de Medicina da Universidade Federal de Pelotas.

\section{Resultados}

A análise dos intervalos de confiança das taxas brutas de prevalências de todas as formas clínicas de tuberculose mostrou oscilações. As taxas brutas apresentaram variação de 51,4 (IC95\% 50,1-52,6) a 74,8 (IC95\%73,4-76,3) nos anos de 2002 e 2003 respectivamente, com tendência ao crescimento de 0,04 ao final do período. Entretanto, nas médias móveis os intervalos de confiança se sobrepunham e as variações percentuais foram de 0,01 (Tabela 1).

As taxas brutas de incidência também variaram no período. A taxa mais baixa foi de 45,8 (IC95\% 44,7-47,0) em 2002, a mais elevada foi 68,6 (IC95\% 67,2-70,0) em 2003, com variações percentuais médias anuais de 0,06 . No mesmo período, as médias móveis e seus intervalos de confiança, não apresentaram variação na incidência da doença (Tabela 2).

Tabela 1. Prevalências de casos de tuberculose em todas formas clínicas (taxas brutas e médias móveis com respectivos intervalos de confiança a 95\%), mudanças percentuais médias anuais. Sapucaia do Sul, RS, 2000-8.

\begin{tabular}{|c|c|c|c|c|}
\hline Ano & No de casos & Taxa bruta $(\text { IC95\%) })^{*}$ & Período & Médias móveis (IC95\%) \\
\hline 2000 & 73 & $59,5(58,1-60,8)$ & $2000-2$ & $58,5(50,8-66,3)$ \\
\hline 2001 & 81 & $64,9(63,5-66,3)$ & 2001-3 & $63,7(55,7-71,8)$ \\
\hline 2002 & 65 & $51,4(50,1-52,6)$ & $2002-4$ & $65,0(56,9-73,0)$ \\
\hline 2003 & 96 & $74,8(73,4-76,3)$ & $2003-5$ & $70,4(62,1-78,7)$ \\
\hline 2004 & 89 & $68,5(67,0-69,9)$ & 2004-6 & $66,8(58,8-74,8)$ \\
\hline 2005 & 91 & $67,9(66,5-69,3)$ & $2005-7$ & $62,3(54,6-69,9)$ \\
\hline 2006 & 87 & $64,0(62,6-65,3)$ & $2006-8$ & $63,5(55,7-71,3)$ \\
\hline 2007 & 76 & $55,1 \quad(53,9-56,3)$ & & \\
\hline 2008 & 91 & $72,2(70,7-73,7)$ & & \\
\hline
\end{tabular}

*Variações percentuais médias anuais: $0,04 .{ }^{* *}$ Variações percentuais médias anuais: 0,01 
Quanto aos desfechos possíveis na situação de encerramento do PMCT no SINAN-SMS não foram observadas diferenças estatisticamente significativas. Os percentuais de cura oscilaram entre 67,2\% em 2002 até 81,7 em 2006. Os percentuais de abandono ao tratamento variaram entre $4,7 \%$ em 2000 a $13,6 \%$ em 2003 e os óbitos entre 2,5\% em 2005 e 19,0\% em 2002 (Tabela 3).

\section{Discussão}

O uso de fontes secundárias para o desenvolvimento de estudos epidemiológicos tem sido questionado pela possibilidade de subnotificação e pelos possíveis erros de classificação. Os dados coletados através do SINAN, em Sapucaia do Sul, aparentemente não apresentaram problemas em relação à completude, consistência, duplicidade e classificação em relação as variáveis pesquisadas ${ }^{21}$.
A taxa média de prevalência encontrada em Sapucaia foi de 64,3 em 100.000 habitantes e a taxa média de incidência foi de 58 casos em 100.000 habitantes que foram consideradas altas. No Brasil a prevalência de casos vem apresentando declínio e a taxa de incidência teve queda de $27 \%$ na última década, conforme os últimos relatórios da Organização Mundial da Saúde ${ }^{5,14}$. Atualmente o Brasil apresenta uma taxa de prevalência em torno de 40 casos em 100.000 habitantes, enquanto que a incidência está em torno de 38 casos em 100.000 habitantes ${ }^{14}$. Fontes estaduais informaram taxas médias que se aproximavam mais das taxas nacionais do que os indicadores encontrados em Sapucaia do Sul. Estudos recentes mostraram que a taxa estadual de incidência em 2008 foi de 44,2 casos por 100.000 habitantes ${ }^{14,22}$. Porto Alegre e a região metropolitana onde o município em estudo se localiza apresentaram as taxas mais elevadas. Nos últimos anos as ta-

Tabela 2. Incidência de tuberculose em todas formas clínicas (taxas brutas e médias móveis com respectivos intervalos de confiança a 95\%), variações percentuais médias anuais. Sapucaia do Sul, RS, 2000-8.

\begin{tabular}{ccccc}
\hline Ano & No de casos & Taxa bruta $\left(\mathbf{I C 9 5 \% ) ^ { * }}\right.$ & Período & Médias móveis $\left(\right.$ IC95\%) $^{* *}$ \\
\hline 2000 & 64 & $52,1(50,9-53,4)$ & $2000-2$ & $53,5(50,8-66,3)$ \\
2001 & 78 & $62,5(61,1-63,9)$ & $2001-3$ & $59,0(55,7-71,8)$ \\
2002 & 58 & $45,8(44,7-47,0)$ & $2002-4$ & $58,5(56,9-73,0)$ \\
2003 & 88 & $68,6(67,2-70,0)$ & $2003-5$ & $63,0(55,1-70,8)$ \\
2004 & 79 & $60,8(59,4-62,1)$ & $2004-6$ & $57,5(58,8-74,8)$ \\
2005 & 80 & $59,7(58,4-61,0)$ & $2005-7$ & $54,7(54,6-69,9)$ \\
2006 & 71 & $52,2(51,0-53,4)$ & $2006-8$ & $57,3(55,7-71,3)$ \\
2007 & 72 & $52,2(51,0-53,4)$ & & \\
2008 & 86 & $68,2(66,8-69,6)$ & & \\
\hline
\end{tabular}

${ }^{*}$ Variações percentuais médias anuais: $0,06 .{ }^{* *}$ Variações percentuais médias anuais: 0,01 .

Tabela 3. Percentuais de cura, de abandono ao tratamento e de óbitos dos casos novos de tuberculose em todas formas clínicas. Sapucaia do Sul, RS, 2000-2008.

\begin{tabular}{lccccc}
\hline \multicolumn{1}{c}{ Ano } & Cura (\%) & Abandono (\%) & Óbitos (\%) & Outros (\%) & Total \\
\hline 2000 & $50(78,1)$ & $3(4,7)$ & $6(9,4)$ & $5(7,8)$ & 64 \\
2001 & $59(75,6)$ & $9(11,5)$ & $5(6,4)$ & $5(6,5)$ & 78 \\
2002 & $39(67,2)$ & $6(10,3)$ & $11(19,0)$ & $2(3,5)$ & 58 \\
2003 & $64(72,7)$ & $12(13,6)$ & $7(8,0)$ & $5(5,7)$ & 88 \\
2004 & $64(81,0)$ & $9(11,4)$ & $5(6,3)$ & $1(1,3)$ & 79 \\
2005 & $62(77,5)$ & $9(11,2)$ & $7(2,5)$ & $2(2,8)$ & 80 \\
2006 & $58(81,7)$ & $4(5,6)$ & $8(11,1)$ & $3(4,2)$ & 71 \\
2007 & $56(77,8)$ & $5(6,9)$ & $8(9,3)$ & $6(7$ & 72 \\
2008 & $58(77,9)$ & $5(5,8)$ & 0,84 & & 86 \\
Valor p & 0,69 & 0,32 & &
\end{tabular}

* Qui-quadrado tendência linear 
xas de prevalência e incidência de Porto Alegre e Região Metropolitana de Porto Alegre estavam acima de 90 e 70 casos por 100.000 habitantes respectivamente ${ }^{22,23}$

Estudo realizado no Brasil analisou a incidência de tuberculose e a taxa de cura ${ }^{24}$ com o objetivo de descrever sua distribuição geográfica, a partir de um conjunto de indicadores epidemiológicos e operacionais de dados de notificação oficial do SINAN. Houve extensas diferenças estaduais em relação à incidência e às categorias de desfecho. Em 2004, o Brasil apresentou taxa de incidência de 41/100.000 habitantes, com 74.540 casos novos notificados. Desses, 52,8\% eram casos pulmonares com baciloscopia positiva, $24,1 \%$ estavam em tratamento supervisionado, 63,5\% eram provenientes de capitais ou das regiões metropolitanas e 54,9\% eram casos curados. A proporção de cura alcançou 72,4\% para casos novos, $47 \%$ para casos novos HIV positivos, $64,9 \%$ para recidivas, $64,5 \%$ transferências e $40 \%$ para reingressos após abandono. A taxa de cura para os casos novos em tratamento supervisionado foi de $77,1 \%{ }^{24}$. Esse estudo indicou o tratamento supervisionado como uma estratégia para o controle da doença.

Outro estudo teve com objetivo analisar o perfil e as tendências da mortalidade por tuberculose no Brasil, de 1980 a 2004. Mostrou redução inconstante do número e da taxa de mortalidade por tuberculose ao longo do período estudado, de 5,8 em 1980 para 2,8 por 100.000 habitantes, em 2004. Foi observada redução acentuada da mortalidade até 1985, mais evidente nos estados do Rio de Janeiro e São Paulo. Entretanto, constatou-se aumento da mortalidade por tuberculose em idades mais avançadas, além disso, segundo os autores, a mortalidade aumentaria quase $50 \%$ se fossem incluídos os óbitos por tuberculose como causa associada e por sequela de tuberculose como causa básica ${ }^{25}$. Este estudo propõe que o Plano Nacional de Controle da Tuberculose deve incorporar ações que se destinem a reduzir os óbitos que ocorrem não somente por causa básica, mas também como causa associada, e considere a tendência de aumento da idade dos pacientes em risco de evoluírem ao óbito ${ }^{25}$. Embora a mortalidade encontrada neste estudo tenha sido menor do que os resultados encontrados em Sapucaia do Sul as metas nacionais também não foram alcançadas.

Em relação aos desfechos possíveis, em Sapucaia do Sul foram encontrados resultados abaixo das metas nacionais, como por exemplo, tratar $100 \%$ dos casos diagnosticados. No acompa- nhamento pelo Programa Municipal de Controle da Tuberculose observou-se que alguns casos foram notificados por ocasião do óbito e não chegaram a iniciar o tratamento assim como foram encontrados abandonos primários, quando pessoas foram diagnosticadas no hospital municipal durante a internação, porém não foram localizadas após a alta. A meta de cura de 85\% dos casos novos com baciloscopia positiva também não foi atingida. $\mathrm{O}$ abandono ao tratamento e o óbito estão dentro de percentuais aceitáveis pelo PNCT, mas acima do que foi pactuado, ou seja, manter em percentuais abaixo de $5 \%$. Também foi observado que alguns casos foram mantidos no SINAN mesmo com a situação de encerramento como mudança de diagnóstico. Em 2000 a cura no Brasil foi de $69 \%$, o abandono do tratamento $13 \%$ e o óbito de $3,9 \%$, enquanto que em 2007 a cura alcançou 70\%, o abandono do tratamento $8 \%$ e os óbitos vêm se mantendo desde 2005 em 2,5\% ${ }^{14,25}$ Em 2008, o Rio Grande do Sul apresentou cura de $66,2 \%$ e os percentuais de abandono ao tratamento e de óbitos em 10,6\% e $9,4 \%$ respectivamente, ou seja, revelaram resultados semelhantes aos do presente estudo ${ }^{22}$.

Em Porto Alegre, RS, foi realizado um estudo com o objetivo de analisar as causas da não adesão ao tratamento; durante três anos 340 pacientes foram atendidos pelo Programa de Controle da Tuberculose. Os resultados mostraram que 245 casos receberam alta, sendo por $68 \%$ por cura, 10,2\% abandonaram o tratamento, 9,0\% foram ao óbito, 8,6\% foram transferidos, os restantes receberam altas por mudança de diagnóstico ou apresentaram falência. Os motivos de abandono registrados nos prontuários e verbalizados pelos pacientes incluíram problemas econômico-financeiros e decorrentes do tratamento da doença ${ }^{26}$. Em Cuiabá (MT) foi realizado estudo para identificar possíveis preditores de abandono ao tratamento. $\mathrm{O}$ estudo mostrou taxa global de abandono de $16,1 \%$. Os resultados indicaram elevada incidência de abandono, sendo considerados como preditores: tratamento não supervisionado, ano de tratamento ${ }^{27}$.

Desta forma, os resultados revelados em Sapucaia do Sul foram considerados insatisfatórios em relação às metas, o que compromete o controle da doença, já que não se consegue interromper a cadeia de transmissão. O presente estudo contribui para confirmar as diferenças regionais na prevalência e incidência da tuberculose, atingindo níveis maiores em estados com mais prevalência de infecção por HIV como no Rio Grande do Sul ${ }^{28}$. Além disso, os achados do pre- 
sente estudo apontaram para inefetividade dos serviços de saúde no acompanhamento e cumprimento das metas preconizadas para o controle da doença. O tempo decorrido entre o diagnóstico e o início do tratamento, bem como a realização do tratamento completo são as bases para o controle da infecção por tuberculose ${ }^{28}$. A inclusão do monitoramento da tuberculose entre as atividades prioritárias da Estratégia Saúde da Família e, consequentemente, com a descentralização do Programa Municipal de Controle da Tuberculose e a ampliação da estratégia DOTS implementados gradativamente no município a partir de $2006^{16}$ poderão contribuir para alcançar o controle da doença

\section{Colaboradores}

MA Heck, JSD Costa e MF Nunes participaram igualmente de todas as etapas de elaboração do artigo.

\section{Referências}

1. Brasil. Ministério da Saúde (MS). Fundação Nacional da Saúde. Tuberculose: Guia de Vigilância Epidemiológica. Brasília: MS; 2006.

2. Brasil. Ministério da Saúde (MS). Cadernos de Atenção Básica. Manual Técnico para o Controle da Tuberculose. Brasília: MS; 2002.

3. Filho AC, Kritski AL, Barreto ÂW, Lemos ACM, Netto AR, Guimarães CA, Silva CL, Sant'Anna CC, Haddad DJ, Lima DS, Matos ED, Melo FCQ, Melo FAF, Gerhardt Filho G, Marsico GA, Silva G, Siqueira HR, Campos H, Saconato H, Dourado I, Rosemberg J, Braga JU, Santos JR, Seiscento M, Conde MB, Dalcolmo MP, Almeida MMB, Penna MLF, Barreto ML, Hijjar MA, Andrade MKN, Cardoso NC, Pineda NIS, Leite OHM, Picon P, Silva RF, Cavalcanti S, Pereira SM, Augusto VM, Galesi V, Pinto WP. II Consenso Brasileiro de Tuberculose: Diretrizes Brasileiras para Tuberculose 2004. Jornal Brasileiro de Pneumologia 2004; 30(Supl. 1):S57-S86.

4. World Health Organization (WHO). La tuberculosis en Barcelona, Programa de prevención y control de la tuberculosis de Barcelona. Barcelona: WHO; 2005.

5. World Health Organization (WHO). Global tuberculosis control: surveillance, planning, financing. Genebra: WHO Report; 2007. (WHO/HTM/TB/ 2007.376). [acessado 2009 nov 30]. Disponível em: www.who.int/tb/publications/global_report/2007/ pdf/full.

6. World Health Organization (WHO). Global tuberculosis control surveillance, planning, financing. Barcelona: Who Report; 2008.

7. Brasil. Ministério da Saúde (MS). Fundação Nacional de Saúde. Centro de Referência Prof. Hélio Fraga. Sociedade Brasileira de Pneumologia e Tisiologia. Controle da Tuberculose - Uma Proposta de Integração Ensino-Serviço. 5a Edição. Rio de Janeiro: MS; 2002. 
8. Brasil. Ministério da Saúde (MS). Departamento de Vigilância Epidemiológica. Plano Nacional de Controle da Tuberculose. Brasília: MS; 2004.

9. Brasil. Ministério da Saúde (MS). Diretrizes operacionais, Pactos pela vida em defesa do SUS e de gestão. Brasília: MS; 2006.

10. Hijjar MA, Procópio MJ, Freitas LMR, Guedes R, Bethlem EP. Epidemiologia da tuberculose: importância no mundo, no Brasil e no Rio de Janeiro. Pulmão 2005; 14(4):310-314.

11. Instituto de Pesquisa Econômica Aplicada (IPEA). Objetivos de desenvolvimento

do milênio: relatório nacional de acompanhamento. Brasília; 2004 [acessado 2007 ago 6]. Disponível em: http://www.pnud.org.br/odm/arquivos/odm_ rna2005.pdf

12. Hijjar AM, Oliveira MJPR, Teixeira GM. A tuberculose no Brasil e no Mundo. Boletim de Pneumologia Sanitária 2001; 9(2):9-14.

13. Barreira D, Grangeiro A. Avaliação das estratégias de controle da tuberculose no Brasil. Rev Saude Publica 2007; 41(supl. 1):4-8.

14. Brasil. Ministério da Saúde (MS). Secretaria de Vigilância em Saúde. Departamento de Vigilância Epidemiológica. Programa Nacional de Controle da Tuberculose PNCT - Brasil. Brasília: MS; 2009.

15. Plano Estratégico Regional para o Controle da Tuberculose 2005-2015. Organização Pan-Americana de Saúde. [acessado 2007 ago 6]. Disponível em: http://www.paho.org/Portuguese/AD/DPC/CD/tbfl yer06port.pdf

16. Sapucaia do Sul. Secretaria Municipal de Saúde. Vigilância em Saúde: Plano Municipal de Controle da Tuberculose. Sapucaia do Sul: Secretaria Municipal de Saúde; 2009.
17. Brasil. Ministério da Saúde (MS). Vigilância Epidemiológica. Tuberculose. Guia da Vigilância Epidemiológica. Brasília: MS; 2002.

18. Toscana CM. Tuberculose. In: Duncan BB, Schmidt MI, Giugliani ERJ. Condutas de Atenção Primaria Baseadas em Evidências. Medicina Ambulatorial. 3a Edição. Porto Alegre: Artmed; 2004. p. 1354-1364.

19. Rosenberg D. Trend Analysis and Interpretation. Key Concepts and Methods for Maternal and Child Health Professionals. Maryland: Division of Science, Education and Analysis Maternal and Child Health Information Resource Center; 1997.

20. Latorre MRDO, Cardoso MRA. Análises de séries temporais em epidemiologia: uma introdução sobre os aspectos metodológicos. Revista Brasileira de Epidemiologia 2001; 4(3):145-152.

21. Brasil. Ministério da Saúde (MS). SINAN; Sistema de informação de agravos de notificação; Normas e rotinas. Brasília: MS; 2007.

22. Rio Grande do Sul. Secretaria Estadual de Saúde. Coordenação Estadual de Vigilância em Saúde. Divisão de Vigilância Epidemiológica. Programa Estadual de Controle da Tuberculose (PECT). Situação atual da tuberculose dados epidemiológicos. Porto Alegre: Secretaria Estadual de Saúde; 2009.

23. Ott WP, Jarczewski CA. Combate à tuberculose sob novo enfoque no Rio Grande do Sul. Boletim Epidemiológico 2007; 9(5):1-7.

24. Bierrenbach AL, Gomes ABF, Noronha EF, Souza MFM. Incidência de tuberculose e taxa de cura, Brasil, 2000 a 2004. Rev Saude Publica 2007; 41(Supl. 1): 24-33.

25. Bierrenbach AL, Duarte EC, Gomes ABF, Souza MFM. Tendência da mortalidade por tuberculose no Brasil, 1980 a 2004. Rev Saude Publica 2007; 41(Supl. 1):15-23. 
26. Mendes AM, Fensterseifer LM. Tuberculose: porque os pacientes abandonam o tratamento? Boletim de Pneumologia Sanitária 2004; 12(1):27-38.

27. Ferreira SMB, Silva AMC, Botelho C. Abandono do tratamento da tuberculose pulmonar em Cuiabá MT - Brasil. Jornal Brasileiro de Pneumologia 2005; 31(5):427-435.

28. Barreto ML, Teixeira MG, Bastos FI, Ximenes RAA, Barata RB, Rodrigues LC. Sucessos e fracassos no controle das doenças infecciosas no Brasil: o contexto social e ambiental, políticas, intervenções necessidades de pesquisa. The Lancet 2011; 47-60.

Artigo apresentado em 31/10/2011

Aprovado em 12/03/2012

Versão final aprovada em 12/04/2011 